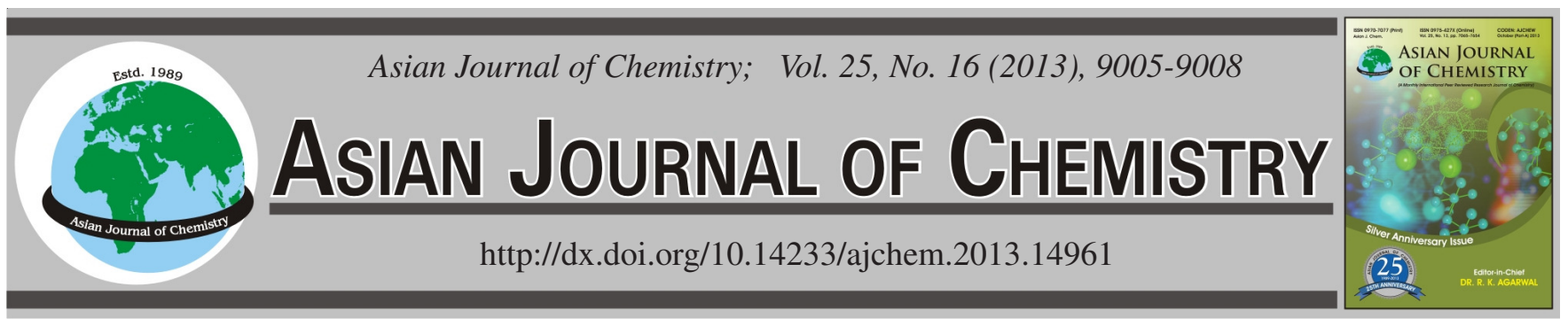

\title{
Monte Carlo Simulation for the Relation Between the Combining Styrene Content and Monomer Conversion in SBR Emulsion Co-Polymerization
}

\author{
YAN-JiAng JiN $^{1,2}$, BEN-XIAN SHEN ${ }^{1}$, LeI YANG ${ }^{2}$ and Ji-GANG ZhaO ${ }^{1, *}$
}

${ }^{1}$ State Key Laboratory of Chemical Engineering, East China University of Science and Technology, Shanghai 200237, P.R. China

${ }^{2}$ Jilin Petrochemical Company, PetroChina, Jilin 132021, P.R. China

*Corresponding author: Fax: +86 21 64252851; Tel: +86 21 64252916; E-mail: zjg@ecust.edu.cn

\begin{abstract}
Monte carlo method has been used to simulate the emulsion co-polymerization of styrene-butadiene-rubber in a micro-reactor of $10^{-20} \mathrm{~m}^{3}$ with the original industry receipe. The growth process of the chain was studied with the assumption of generation one radical and one chain. The results showed that the instantaneous combining styrene content was not ascertained, but it would be oscillated in some range in one monomer conversion. The overall trend was that the instantaneous combining styrene content remain unchanged in the main with the rise of monomer conversion in the preliminary stage and then it increased. The raising rate of monomer conversion would become higher in the later stage of the reaction. The average combining styrene content went up with the rise of monomer conversion. When the monomer conversion was between $38 \%$ and $74 \%$, the combining styrene content in the styrene-butadiene-rubber could meet the national specified quality that the combining styrene content between $22.5 \%$ and $24.5 \%$.
\end{abstract}

Key Words: Monte carlo method, Combining styrene content, Monomer conversion, Styrene butadiene rubber.

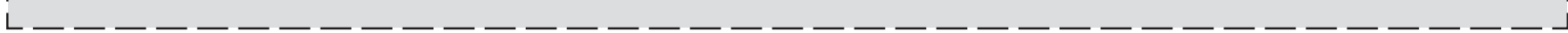

\section{INTRODUCTION}

The experimental studies of styrene-butadiene copolymer emulsion of styrene-butadiene-rubber have been conducted for many years and a lot of relevant data has been achieved. As it required data to describe the co-polymerization process, samples must be taken out from the reaction and then tested by NMR, IR and other equipment to get various parameters of the co-polymerization process. But it would affect the co-polymerization process and also produce error if too much samples were obtained. So it could not completely reflect the alteration of parameters of the reaction just by experimental data. It is time-consuming. So it need simulate the co-polymerization process to get various parameters ${ }^{1,2}$. The emulsion co-polymerization process is a random process, so it could be studied by Monte-Carlo simulation ${ }^{3}$.

Compared with Monte-carlo simulation, other mathematical models and computer simulations need complex mathematics. The convergence of model equations, especially differential equation is difficult to judge and then it is difficult to solve. While the Monte-carlo method is simple in principle, simple in programming and easy to understand. Therefore, in this paper, Monte-carlo method was used to simulate the relationship of the combining styrene content and monomer conversion. The variation of the combining styrene content in styrene- butadiene-rubber with different monomer conversion was studied. It would provide the basic data and theoretical basis for increasing the monomer conversion in styrene-butadienerubber emulsion polymerization.

\section{EXPERIMENTAL}

Principles of simulation: For the styrene-butadiene co-polymerization process, at the beginning of the reaction, styrene monomer and butadiene monomer were respectively set as $\mathbf{M}_{1}, \mathbf{M}_{2}$ and monomer radicals were $\mathbf{M}_{1}, \mathbf{M}_{2}$, based on the polymer theory ${ }^{4}$ :

$$
\begin{aligned}
& \sim M_{1}+\left\{\begin{array}{lll}
M_{1} & k_{11} \sim \sim M_{1} \cdot & R_{11}=K_{11}\left[M_{i}\right]\left[M_{1}\right] \\
M_{2} & \stackrel{k_{12}}{\longrightarrow} \sim M_{2} & R_{12}=K_{12}\left[M_{1}\right]\left[M_{2}\right]
\end{array}\right. \\
& \Omega M_{2}+ \begin{cases}M_{1} \stackrel{k_{21}}{\sim} \sim M_{1} \cdot R_{21}=K_{21}\left[M_{2}\right]\left[M_{1}\right] \\
M_{2} \stackrel{k_{22} \sim}{\sim M_{2}} \cdot R_{22}=K_{22}\left[M_{2}\right]\left[M_{2}\right]\end{cases}
\end{aligned}
$$

where $\mathrm{R}_{\mathrm{i}, \mathrm{j}}$ was the chain growth rate, $\mathrm{k}_{\mathrm{i}, \mathrm{j}}$ was the rate constant, $\left[\mathrm{M}_{\mathrm{i}}\right]$ was the concentration of monomer $\mathrm{i},\left[\mathrm{M}_{\mathrm{i}}\right]$ was the radical concentration of monomer $\mathrm{i}$. $(\mathrm{i}, \mathrm{j}=1,2)$. The reactivity ratio of styrene was $r_{1}=k_{1,1} / k_{1,2}=0.64^{5}$, but the butadiene was $r_{2}=k_{2,2} / k_{2,1}$ $=1.44^{5}$. 
Radical $\left[\mathrm{M}_{1}\right.$. adding by monomer $\mathrm{M}_{1}$ or $\mathrm{M}_{2}$ is a pair of competitive reactions [eqn. (1) and (2)], the probability of formation of $\mathrm{M}_{1} \mathrm{M}_{1}$ and $\mathrm{M}_{1} \mathrm{M}_{2}$ was:

$$
\begin{gathered}
\mathrm{p}_{11}=\frac{\mathrm{R}_{11}}{\mathrm{R}_{12}}=\frac{\mathrm{r}_{1}\left[\mathrm{M}_{1}\right]}{\mathrm{r}_{1}\left[\mathrm{M}_{1}\right]+\left[\mathrm{M}_{2}\right]} \\
\mathrm{p}_{12}=\frac{\mathrm{R}_{12}}{\mathrm{R}_{11}+\mathrm{R}_{12}}=\frac{\left[\mathrm{M}_{2}\right]}{\mathrm{r}_{1}\left[\mathrm{M}_{1}\right]+\left[\mathrm{M}_{2}\right]}
\end{gathered}
$$

Obviously, $\mathrm{P}_{11}=1-\mathrm{P}_{12}$. And the probability of formation of $\mathrm{M}_{2} \mathrm{M}_{2}$ and $\mathrm{M}_{2} \mathrm{M}_{2}$ was:

$$
\begin{aligned}
& \mathrm{p}_{22}=\frac{\mathrm{R}_{22}}{\mathrm{R}_{21}+\mathrm{R}_{22}}=\frac{\mathrm{r}_{2}\left[\mathrm{M}_{2}\right]}{\left[\mathrm{M}_{1}\right]+\mathrm{r}_{2}\left[\mathrm{M}_{2}\right]} \\
& \mathrm{p}_{21}=\frac{\mathrm{R}_{21}}{\mathrm{R}_{21}+\mathrm{R}_{22}}=\frac{\left[\mathrm{M}_{1}\right]}{\left[\mathrm{M}_{1}\right]+\mathrm{r}_{2}\left[\mathrm{M}_{2}\right]}
\end{aligned}
$$

$\mathrm{P}_{22}=1-\mathrm{P}_{21}$

Simulation assumption: The styrene-butadiene emulsion co-polymerization is in line with the classic Smith-Ewart theory ${ }^{6}$ and some reasonable assumptions for the polymerization in order to simplify the model are as follows:

(1) The initiator produced only one free radical and then into one micelle to generate one particle. One chain generated in the particle and the reaction conducted with monomers coming from the monomer drops adding into the chain. Chain transfer and termination were not considered.

(2) The mass fraction of styrene in the monomer drops and the mass fraction of styrene of monomer in the particle was equal.

Mathematical modeling and the simulation methods: The emulsion co-polymerization proceeded in a micro-reactor of $10^{-20} \mathrm{~m}^{3}$. In the micro- reactor the process of generating of a particle and the changes in the particle were all detailed. The monomer conversion, the average combining styrene content and the instantaneous combining styrene content were all recorded. Fig. 1 shows the computer simulation flowchart of emulsion polymerization. The ratio of styrene and butadiene was in line with the standard formula. The density of styrene monomer, butadiene monomer and polymer were respectively $0.903,0.855,0.930\left(\mathrm{~g} / \mathrm{cm}^{3}\right)$.

There were two cases in the computer simulation of styrenebutadiene co-polymerization process. When the end of the free radical chain was $\mathrm{M}_{1}$, a random number $\mathrm{r}$ generated. When $\mathrm{r}>\mathrm{P}_{11}, \mathrm{M}_{1}$ added to the end of the free radical and formed $\mathrm{M}_{1} \mathrm{M}_{1}$. When $\mathrm{r}<\mathrm{P}_{11}, \mathrm{M}_{2}$ added to the end of the free radical and formed $\mathrm{M}_{1} \mathrm{M}_{2}$. When the end of the free radical chain was $\mathrm{M}_{2}$, a random number $\mathrm{r}$ generated. When $\mathrm{r}>\mathrm{P}_{21}, \mathrm{M}_{1}$ added to the end of the free radical and formed $\mathrm{M}_{2} \mathrm{M}_{1}$. When $\mathrm{r}<\mathrm{P}_{22}$, $\mathrm{M}_{2}$ added to the end of the free radical and formed $\mathrm{M}_{2} \mathrm{M}_{2}$. The generation of random number was in accordance with mixed congruential method ${ }^{7}$.

The syntax for mixed congruential method in MATLAB programming was MixMOD.

$$
\text { Call format: } \mathrm{r}=\operatorname{MixMOD}\left(\mathrm{x}_{0}, n \text {, type }\right)
$$

where $\mathrm{x}_{0}$ was random seed, $n$ was the number of the random number, type was the form of formula of mixed congruential method, $r$ was random number sequence.

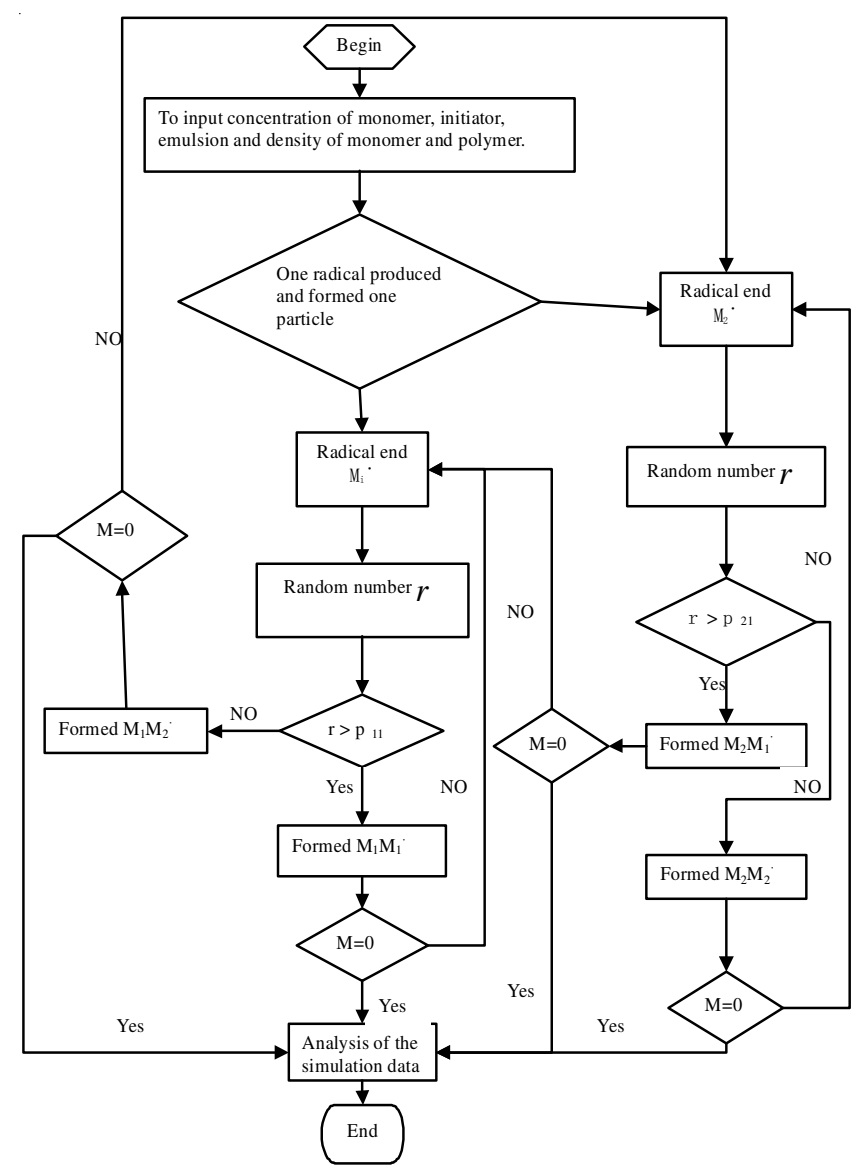

Fig. 1. Computer simulation flow chart of emulsion polymerization

Evaluation index: (1) Monomer conversion C: The mass ratio of monomer consumed with amount of the initial monomer.

$$
\mathrm{C}=\frac{\mathrm{M}_{0}-\mathrm{M}}{\mathrm{M}_{0}}
$$

(2) Average combining styrene content $f_{1}$ : The mass ratio of monomer consumed in a moment with polymer.

$$
\mathrm{f}_{1}=\frac{\mathrm{M}_{1}}{\mathrm{M}_{1}+\mathrm{M}_{2}}
$$

(3) Instantaneous combining styrene content $F_{1}$ : In the simulation, the instantaneous combining styrene content in one point was $0.17 \%$ of the total number before this point, in this period the mass ratio of styrene consumed with the initial styrene.

$$
F_{1}=\frac{d\left[M_{1}\right]}{d\left[M_{1}\right]+d\left[M_{2}\right]}
$$

where $\mathrm{M}_{0}$ was the mass of the initial monomer, $\mathrm{g}$; $\mathrm{M}$ was the mass of remaining styrene, $\mathrm{g} ; \mathrm{M}_{1}$ was the mass of styrene consumed, $\mathrm{g} ; \mathrm{M}_{2}$ was the mass of butadiene consumed, $\mathrm{g}$; $\mathrm{d}\left[\mathrm{M}_{1}\right], \mathrm{d}\left[\mathrm{M}_{2}\right]$, respectively were $0.17 \%$ of total number the styrene and butadiene consumed in one point, $\mathrm{g}$.

\section{RESULTS AND DISCUSSION}

The relationship of styrene content in remaining monomer with monomer conversion. 
When the mass ratio of styrene and butadiene was 72/28 in the styrene-butadiene-rubber emulsion co-polymerization (Fig. 2), with the reaction proceeding, the monomer conversion continuously rose and the mass ratio of styrene in the remaining monomer gradually increased. In the early stage of polymerization reaction, the growth of the remaining styrene content was slow. While in the late stage of polymerization reaction, the styrene content in polymer increased rapidly.

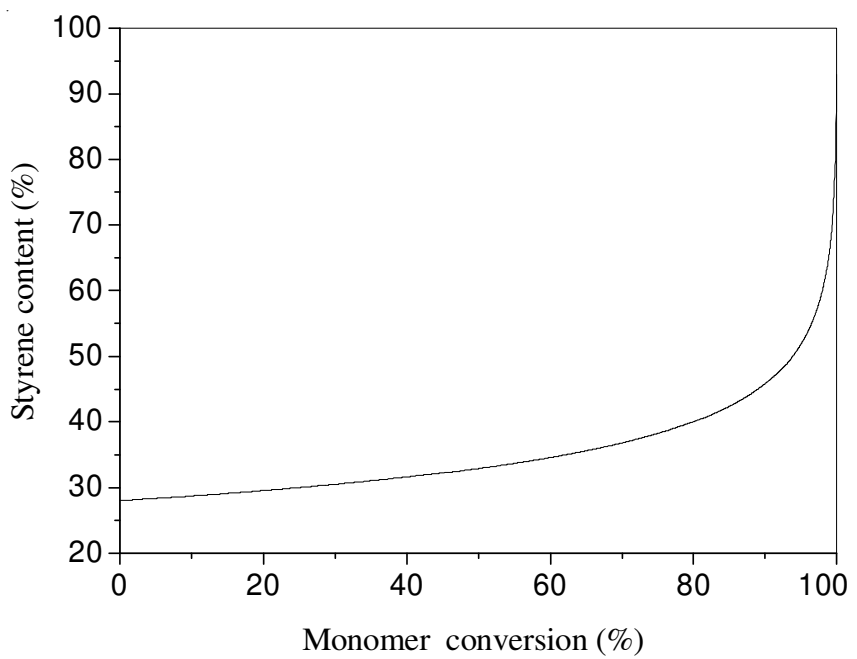

Fig. 2. Evolution of styrene content with monomer conversion in surplus monomer

The relationship of the instantaneous combining styrene content with monomer conversion.

The relationship of the instantaneous combining styrene content with monomer conversion was shown in the left figure of Fig. 3, while the right was the cubic spline fitting of the instantaneous styrene content. From the left figure, the instantaneous styrene content waved in $(21 \pm 1) \%$ in the beginning of the reaction. When the monomer conversion reached $62 \%$, the instantaneous styrene content waved in $(27 \pm 1) \%$. When the monomer conversion reached $74 \%$, the instantaneous styrene content waved in $(30 \pm 1) \%$. It showed that the instantaneous styrene content was uncertain under one monomer conversion, but waved in a range of changes. From the right figure, the overall trend of the instantaneous styrene content was that with the monomer conversion increasing it kept the same and then it increased with the monomer conversion. In the standard formula, the instantaneous styrene content was about $22.0 \%$ in the beginning of the reaction. When the monomer conversion reached $62 \%$ and $74 \%$, the instantaneous styrene content respectively were $27.6 \%$ and $29.6 \%$.

The relationship of the change rate of instantaneous combining styrene content with monomer conversion.

The derivation of Fig. 3 to the monomer conversion and got the relationship shown in Fig. 4. From the figure, when the conversion reached $32 \%$, the curve increased obviously. And the instantaneous styrene content increased obviously. When the monomer conversion reached $62 \%$, the rate of change of the instantaneous styrene content was 0.4005 ; When the monomer conversion reached $74 \%$, it was 0.6753 .

The relationship of the average combining styrene content with monomer conversion.
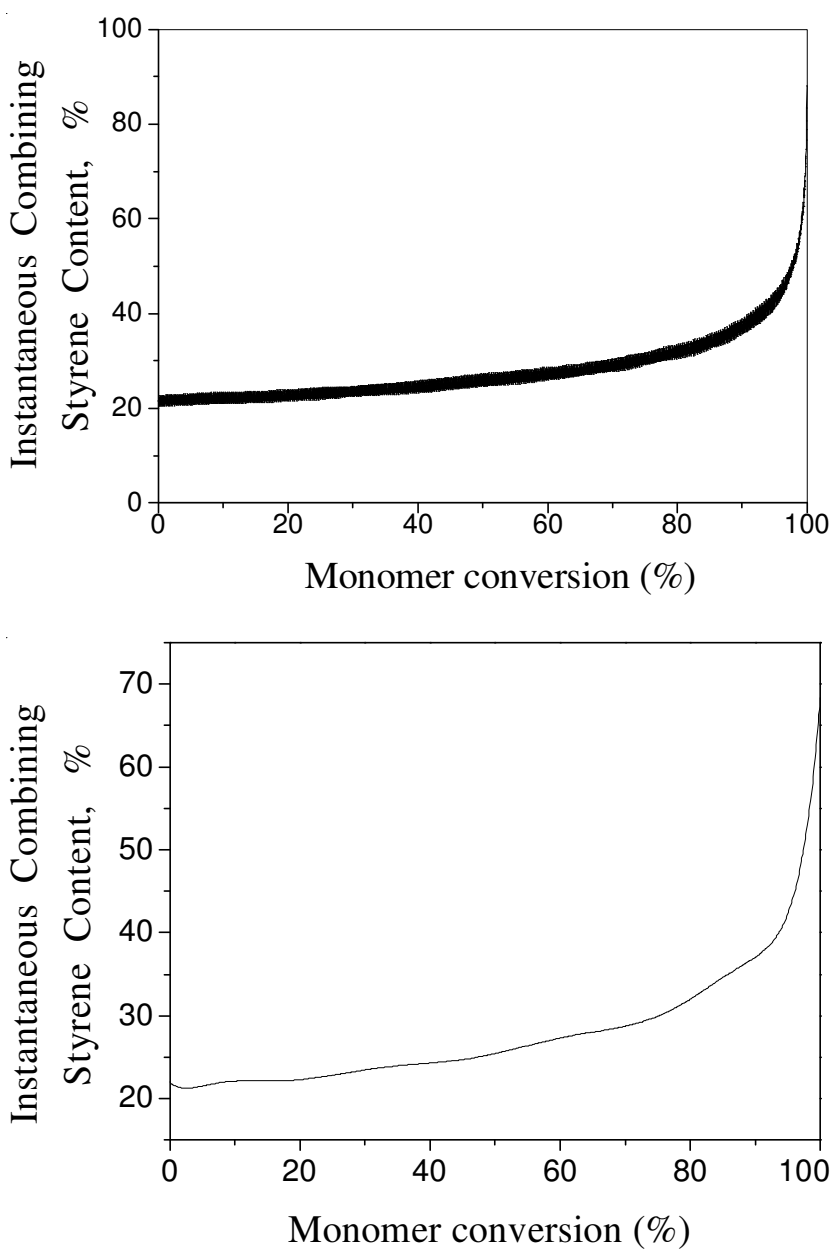

Fig. 3. Evolution of instantaneous combining styrene content with monomer conversion

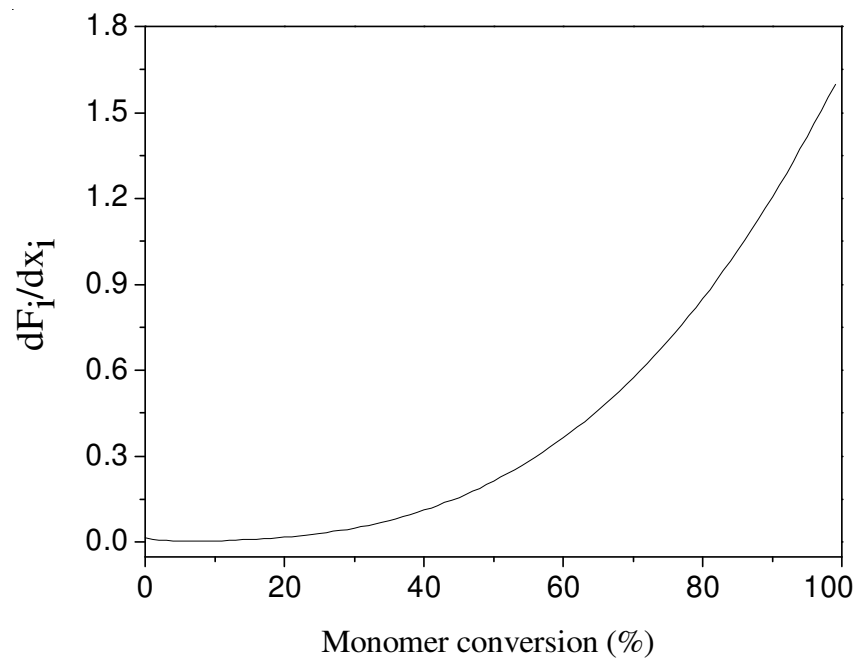

Fig. 4. Evolution of instantaneous combining styrene rate with monomer conversion

The relationship of the average styrene content with the monomer conversion was shown in Fig. 5. From the figure, the average styrene content increased with the monomer conversion becoming higher. When the monomer conversion of ESBR was between $38 \%$ to $74 \%$, the average styrene content was $22.5 \%$ to $24.5 \%$ and it was in line with the national specified quality (GB8655-88). 


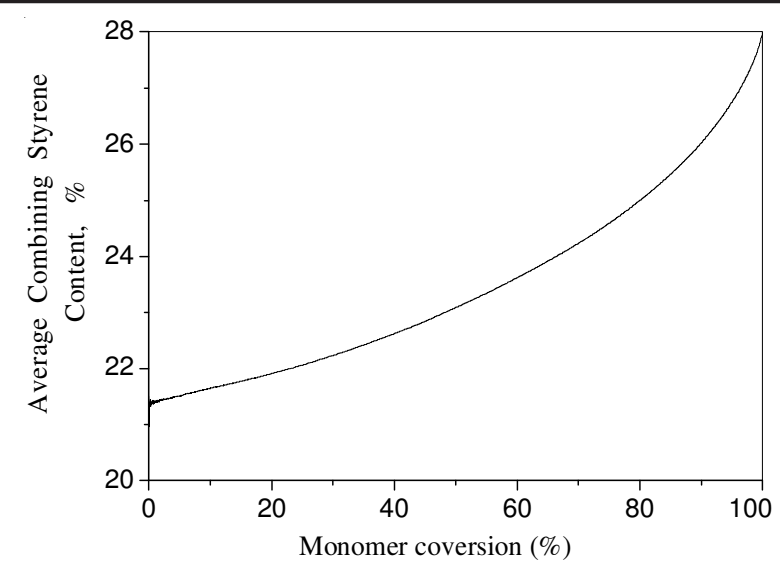

Fig. 5. Evolution of average combining styrene content with monomer conversion

\section{Conclusions}

In the original industrial receipe of emulsion co-polymerization of styrene-butadiene-rubber, the process of one free radical and growth of a chain in a micro-reactor of $10^{-20} \mathrm{~m}^{3}$ was simulated by Monte-Carlo method. The results showed that:

(1) As the reaction proceeded, monomer conversion rose, the mass ratio of styrene of remaining monomer increased.
And the mass ratio of styrene of remaining monomer increased faster in the later stage of emulsion polymerization.

(2) The instantaneous styrene content was uncertain under one monomer conversion, but waved in a range of changes. The overall trend of the instantaneous styrene content was that with the monomer conversion increased it kept the same and then it increased with the monomer conversion.

(3) The average styrene content increased with the monomer conversion becoming higher. When the monomer conversion of ESBR was between $38 \%$ to $74 \%$, the average styrene content was $22.5 \%$ to $24.5 \%$ and it was in line with the national specified quality (GB8655-88).

\section{REFERENCES}

1. J.H. Huang and S.K. Jiao, China Synth. Rubber Ind., 20, 265 (1997)

2. F. Gao and A. Penlidis, Proc. Polym. Sci., 27, 403 (2002),

3. H.X. Huang, Acta Sci. Nat. Univ. Norm Hunan, 6, 2 (1996).

4. Z.R. Pan, Polymer Chemistry, Chemical Industry Press, Beijing, p. 168 (2003).

5. J.H. Huang and S.K. Jiao, China Elastomerics, 7, 156 (1997).

6. T.Y. Cao, Q.P. Liu and J.S. Hu, Polymer Emulsion Polymerization Theory and Application Performance, Chemical Industry Press, BeiJing, 180 (1997).

7. C. Gong and Z.L. Wang, MATLAB Algorithm Commonly Used Procedures Set, Publishing House of Electronics Industry, Beijing, p. 145 (2011). 